\title{
Combining Ability for Drought Tolerance in Upland Rice Varieties at Reproductive Stage
}

\author{
G. M. Malemba ${ }^{1}$, F. M. Nzuve ${ }^{1}$, J. M. Kimani ${ }^{2}$, M. F. Olubayo ${ }^{1} \&$ J. W. Muthomi ${ }^{1}$ \\ ${ }^{1}$ Department of Plant Science and Crop Protection, University of Nairobi, Nairobi, Kenya \\ ${ }^{2}$ Kenya Agricultural and Livestock Research Organization (KALRO), Industrial Crops Research Centre, Mwea, \\ Kerugoya, Kenya \\ Correspondence: G. M. Malemba, Department of Plant Science and Crop Protection, University of Nairobi, P.O. \\ Box 29053-0625, Nairobi, Kenya. E-mail: malembageoffrey@gmail.com
}

Received: November 20, 2016

Accepted: January 13, 2017

Online Published: February 15, 2017

doi:10.5539/jas.v9n3p138

URL: http://dx.doi.org/10.5539/jas.v9n3p138

\begin{abstract}
Rice is an important food crop for human population ranking second among the mostly consumed cereal grains worldwide. Upland rice production is greatly constrained by drought stress resulting from rainfall variation patterns. Cultivation of drought tolerant varieties is considered the best option for drought management in rice production. The already released upland rice varieties are drought susceptible and have poor grain attributes hence, the aim of this study was to determine the combining ability for drought tolerance in upland rice. Four upland NERICA and two upland rice varieties were selected as parents for generating $\mathrm{F}_{1} \mathrm{~s}$ crosses following $6 \times 6$ complete diallel. The generated $30 \mathrm{~F}_{1}$ crosses were advanced to $F_{2}$ population for field evaluation. The $F_{2}$ progenies together with six parents were planted in two sites; KALRO-Mwea Center Farm and Kirogo research Farm following a randomized complete block design in three replications. Drought stress was initiated 45 days after sowing after which data was collected on drought and agronomic parameters. The study revealed large genetic variations among the genotypes used. Both GCA and SCA were significant indicating the importance of both additive and non additive gene action in the expression of studied traits. In this study NERICA 2 and NERICA 15 were identified as good combiners for drought tolerance and grain yield under drought conditions. The single crosses namely; NERICA $15 \times$ NERICA 2, NERICA $1 \times$ NERICA 15, NERICA $11 \times$ NERICA 15 and NERICA $2 \times$ NERICA 15 were identified as superior for improving yield under drought conditions.
\end{abstract}

Keywords: combining ability, drought tolerance, rice (Oryza sativa)

\section{Introduction}

Rice is an important commodity and major food source for more than half of the world's population. With the ever increasing population, rice production must be increased by about $40 \%$ by 2025 to satisfy the growing demand without adversely affecting the resource base (Yogameenakshi et al., 2015). In Kenya, rice expansion and production in rainfed upland ecosystems is majorly constrained by abiotic and biotic stresses such as drought, low temperatures in highlands areas, low soil fertility, salinity and diseases such as rice blast (Menge et al., 2013). Following the recent climate variability and competing water for irrigation and other uses, rice production and expansion is likely to be more severely affected by drought. Rice succumbs to drought much faster than other cereals as it requires anaerobic conditions to complete its life cycle (Kumar et al., 2014). Thus, water shortage around flowering and grain filling stage reduces yield drastically as this affects various physiological mechanisms such as floral fertility in rice which is extremely sensitive to water stress (Boonjung \& Fukai 2003; Kumar et al., 2014). Mitigation of drought by application of irrigation may be a more sustainable way for drought improvement though, this may not be effective since, rice irrigation is dependent on rainfall and in years of low rainfall, water supply is limited (Kimani, 2010). Consequently development of drought tolerant cultivars may effectively address the problem of frequent drought in rainfed upland ecosystem (Verulkar et al., 2010) because the crop provides huge opportunity to breed for drought tolerance due to its inherent capacity and wider adaptation in varied environments (Suresh et al., 2013).

Breeding for drought tolerance requires clear understanding of gene action and combining ability of the drought traits and yield components under water stress and non stress conditions. Given that, the success of any plant breeding programme fundamentally depends not only on selection of parents but also breeding methods (Can et 
al., 1997; Torres \& Geraldi, 2007). Therefore, appropriate breeding methodology should be devised. The knowledge of combining ability is useful to assess nicking ability among genotypes and at the same time explicate the nature and magnitude of gene actions involved (Dar et al., 2014). Diallel (Griffing, 1956a, 1956b) and line $\times$ tester (Kempthorne, 1956) mating designs provide dependable information about the general and specific combining ability (GCA and SCA) of parents and their cross combinations and are helpful in estimating various types of gene actions (Verma, 2003).

Many previous studies showed that rice drought traits, rice yield related traits (tiller number, filled grains per panicle and grain weight) and agronomic characters (plant height and days to flowering) are inherited quantitatively and related genetically to one another and influenced by growing environments (Kobayashi et al., 2003). This necessitates the use of multi environmental trials for effective selection of promising lines. Therefore in the present study six parents with different tolerance to water stress were crossed in full diallel to assess the nature of gene action and combining ability for drought traits under drought conditions and non drought conditions and to identify the best combining parents as well as cross combinations for developing drought tolerant upland rice varieties in Kenya.

\section{Materials and Methods}

\subsection{Germplasm}

The germplasm consisted of four upland NERICA varieties and two upland rice varieties which were selected based on their drought tolerance, high yielding, disease tolerance (Rice blast) and good grain quality with aroma (Table 1).

Table 1. Rice genotypes used in the study

\begin{tabular}{lllll}
\hline Variety & Source & Days to Maturity & GY (T/Ha) & Special Attributes \\
\hline NERICA 1 & KALRO* Mwea \& Kibos & $90-100$ & 4.5 & $\begin{array}{l}\text { Aromatic, Blast tolerant, Long grains, Susceptible to } \\
\text { drought }\end{array}$ \\
NERICA 2 & KALRO Mwea & 115 & 6.5 & $\begin{array}{l}\text { Non Aromatic and Drought tolerant } \\
\text { Non Aromatic, Long grains, Tolerance to blast and } \\
\text { NERICA 11 }\end{array}$ \\
& KALRO Mwea \& Kibos & $90-105$ & 7 & $\begin{array}{l}\text { susceptible to drought. } \\
\text { Drought tolerant and Non Aromatic, }\end{array}$ \\
NERICA 15 & KALRO Mwea & 110 & 8 & $\begin{array}{l}\text { Aromatic, high yield ,Susceptible to drought } \\
\text { SARO 5 (TXD 306) }\end{array}$ \\
Komboka & KALRO Mwea & 120 & 8.22 & $\begin{array}{l}\text { High yielding, mild aroma, tolerant to most diseases, } \\
\text { Local adapted cultivar with good grain quality. }\end{array}$ \\
\hline
\end{tabular}

Note. KALRO: Kenya Agricultural Livestock and Research Organization.

Source: National crop variety list (KEPHIS, 2015).

\subsection{Experimental Sites}

Field experiments were conducted in Upland rainfed ecology during February to June 2016 at two different locations namely the KALRO Industrial Crop Research Center (ICRC) Farm Mwea and Kirogo Farm in Kirinyaga County, Mwea Sub-County, in Central Province (Table 2).

Table 2. Description of locations for experimental sites in KALRO Mwea and Kirogo Farm

\begin{tabular}{lll}
\hline \multirow{2}{*}{ Characteristics } & \multicolumn{2}{c}{ Location } \\
\cline { 2 - 3 } & KALRO Center & Kirogo Farm \\
\hline Latitude & $00^{\circ} 32^{\prime} \mathrm{S}$ & $00^{\circ} 38^{\prime} \mathrm{S}$ \\
Longitude & $37^{\circ} 27^{\prime} \mathrm{E}$ & $37^{\circ} 22^{\prime} \mathrm{E}$ \\
Elevation (Meters above sea level) & 1159 & 1150 \\
Annual rainfall $(\mathrm{mm})$ & 850 & 850 \\
Annual maximum Temperature $\left({ }^{\circ} \mathrm{C}\right)$ & 28.6 & 28.6 \\
Annual minimum Temperatures $\left({ }^{\circ} \mathrm{C}\right)$ & 15.6 & 15.6 \\
Soil Type & Nitosol & Vertisols \\
Soil PH & 5.65 & 5.07 \\
\hline
\end{tabular}

Source: Soil analysis result by National Agriculture Research Laboratories (NARL). 


\subsection{Development of Crosses}

In season 1 (January-December, 2015), six parents were grown in hybridization nursery at KALRO Mwea. The susceptible parents were crossed with the tolerant parents in a full diallel, thus producing $30 \mathrm{~F}_{1}$ progenies and six selfed $F_{1}$ progenies. Emasculation of female parents was done using a vacuum emasculator machine that removes anthers from spikelets using suction pressure at $15 \mathrm{kPa}$. All the $\mathrm{F}_{1}$ seeds generated from crosses were then planted in soil boxes at KALRO Center Hybridization Nursery. The panicles were covered with paper bags to prevent extraneous sources of pollen from getting to the pollinated plants. The mature seeds were harvested after attaining physiological maturity and dried up to $14 \%$ moisture content before planting in the field for drought evaluation.

\subsubsection{Experimental Layout, Design and Crop Husbandry}

During second season which was February 2016 to June 2016, the $F_{2}$ seeds and their six parents were planted in two sites in the field using randomized complete block design with three replications to screen them for tolerance to water stress and grain quality. Each genotype was grown in three row plots of $3 \mathrm{~m}$ length with inter row spacing of $20 \mathrm{~cm}$ and intra row spacing of $20 \mathrm{~cm}$. The genotypes were grown in two sets of experiments namely; drought experiment and Non drought experiment (control). Diamonium phosphate (DAP) as a source of P was applied during planting at recommended rate of $60 \mathrm{~kg} \mathrm{P} \mathrm{ha}^{-1}$. Thinning and gaping was carried out at 3-4 leaf stage to maintain single plant per hill at a spacing of $20 \mathrm{~cm}$. The Calcium ammonium nitrate CAN as source of N was top dressed at the rate of $120 \mathrm{~kg} \mathrm{~N} \mathrm{ha}^{-1}$ applied in three splits of $40 \mathrm{~kg} \mathrm{ha}^{-1}$ at 21 days after transplanting, tillering stage and at panicle initiation stage. Rice stem borer were effectively controlled using a synthetic pyrethroid. Three manual weeding were carried out at 20, 40, and 60 days after sowing. Harvesting was carried out manually.

\subsubsection{Drought Experiment}

Crops were watered twice a week and maintained at field capacity ranging between $0 \mathrm{kPa}$ to $-20 \mathrm{kPa}$ until 45 Days after Sowing (DAS). At 45 DAS, stress was initiated by withholding irrigation and protecting the trial from rainfall by covering it with a rainout shelter. Plots were irrigated only when the soil water tension fell below -70 $\mathrm{kPa}$ at $30 \mathrm{~cm}$ soil depth. At this soil water potential, most lines wilted and exhibited leaf rolling and drying. This type of cyclic stress is considered to be efficient in screening for drought tolerance in populations consisting of genotypes with a broad range of growth duration and it ensures that, all lines receive adequate stress during reproductive development (Lafitte et al., 2004).

\subsubsection{Non-Drought Experiment}

From planting to physiological maturity, each plots received water twice per week. Thereafter, watering was done once per week to allow the plants to dry up for harvesting. Tensiometer was installed in all the three replications to monitor soil moisture that was maintained at field capacity ranging between 0 to $-20 \mathrm{kPa}$ throughout the growing period of the crop.

\subsection{Data Collection}

The standard Evaluation System (SES) for rice manual (IRRI, 2014) was used for all traits measured except where stated otherwise. Ten plants were randomly selected and tagged for data collection. One each plant, data were collected on:

$>$ Days to $50 \%$ flowering (DTF) was recorded when $50 \%$ of the panicles of the plants of each plot had all anthers exerted.

> Chlorophyll content $(\mathrm{Cc})$ was measured using SPAD meter at three different places in selected plants at 60, 75 and 90 days then averaged to express in lux units.

$>$ Leaf rolling (LR) by measuring the drought response level on plant leaves which responded to $-70 \mathrm{kPa}$ water potential measured by a $30 \mathrm{~cm}$ underground installed tensiometers. When leaves completely curled in an $\mathrm{O}$ shapeleaf rolling was scored on a scale of 0 to 9: (IRRI, 2014) where; 0 - Leaves healthy; 1 - Leaves start to fold (shallow); 3 - Leaves folding (deep V-shaped); 5 - Leaves fully capped (U-shaped); 7 - Leaf margins touching (0-shape) and 9 - leaves tightly rolled.

$>$ Leaf drying (LD) was scored at the end of the stress period in the morning. A scale of 0 to 9 was used based on a scale (IRRI, 2014). Where; 0 - No symptoms; 1 - Slightly tip drying; 3 - Tip drying extended up to $1 / 4$ length in most leaves; 5 - One forth to $1 / 2$ of all leaves dried; 7 - More $2 / 3$ of all leaves fully dried and 9 - All plants apparently dead. 
> Spikelet fertility (SF) was determined as described by Lafitte et al. (2003). Seven panicles were randomly selected from each plot. The sample was dried and weighed using digital electronic balance, hand threshed and filled and unfilled spikelets were separated. Then filled grains and unfilled spikelets were counted.

The percentage fertile spikelet was calculated by the formula:

$$
\text { Spikelet Fertility }(\%)=100 \times \frac{\text { Number of filled grains in the sample }}{\text { Number of filled grains }+ \text { Number of unfilled spikelets }}
$$

$>$ Days to maturity (DTM) were recorded as the number of days from planting to when $85 \%$ of the panicles in a plant were mature.

$>$ Number of tillers per hill $(\mathrm{NTL} / \mathrm{H})$ was recorded by counting the number of productive tillers per hill.

$>$ Panicle length (PL) was measured at maturity stage.

$>$ Panicle Weight $(\mathrm{PW})$ of the seven randomly harvested panicles at maturity stage was weighed by electronic weighing balance.

$>$ Number of grains per panicle $(\mathrm{NG} / \mathrm{P})$ was obtained from the difference between the total number of spikelets and unfilled spikelets.

$>$ Grain yield $\left(\mathrm{kg} \mathrm{ha}^{-1}\right)$ Harvesting for GY was done at physiological maturity. Samples were harvested and dried in oven to $14 \%$ moisture before weighing, and weight converted to $\mathrm{kg} \mathrm{ha}^{-1}$ then $\mathrm{tha}^{-1}$.

$>$ Thousand grain weight (TGW) a thousand grains dried at $14 \%$ moisture content were weighed by electronic weighing balance.

\subsection{Data Analysis}

\subsubsection{Analysis of Variance}

The data was subjected to analysis of variance using Restricted Maximum Likelihood (REML) in GENSTAT $15^{\text {th }}$ edition. Separation of genotype means was done by using the Fishers protected Least Significant Difference (LSD) at $5 \%$ level.

\subsubsection{Combining Ability Estimates}

The combining ability analysis was carried out as per Griffing's (1956a); Method 1, model I, in SAS Program 9.2 version. The replication and the blocks within replication were random while genotype was the fixed term. Means from REML analysis were used in a linear model regression to estimate GCA and SCA effects.The estimates of GCA and SCA of parents and progenies were calculated as:

$$
\begin{gathered}
\text { GCA effect }\left(\mathrm{g}_{\mathrm{i}}\right)=\frac{1}{n(n-2)}\left[n x_{i}-2 x \ldots\right] \\
\text { SCA effect }\left(\mathrm{S}_{\mathrm{i}}\right)=x_{i j} \frac{1}{n-2}\left(x_{i}+x_{j}\right)+\frac{2}{(n-1)(n-2)} x
\end{gathered}
$$

Where, $x_{i}, x_{j}=$ means of the $i^{\text {th }}$ and $j^{\text {th }}$ parents, respectively; $x . .=$ grand mean; $n=$ number of parent lines.

\subsubsection{Combining Ability Estimates}

The relative importance of GCA and SCA were estimated using the general predicted ratio (GPR) for the traits observed (Baker, 1978). The ratio was estimated as follows,

$$
\frac{2 \sigma_{\mathrm{GCA}}^{2}}{2 \sigma_{\mathrm{GCA}}^{2}+\sigma_{\mathrm{SCA}}^{2}}
$$

Where, $2 \sigma_{\mathrm{GCA}}^{2}$ and $\sigma_{\mathrm{SCA}}^{2}$ are the variance components for GCA and SCA respectively estimated from Griffing's method 1 model I (fixed effects). Ratios close to one indicate additive effects and are important in the inheritance of the trait while ratios close to zero indicate dominance and epistasis effects which are important in the inheritance of the corresponding traits (Peyman et al., 2012).

\section{Results}

\subsection{The Analysis of Variances under Drought Stress and Non-Drought Stress Conditions}

Under drought environment, the mean squares showed that genotypes were highly significant at $\mathrm{P} \leq 0.05$ for all characters. For environment $\times$ genotypes effects the mean square was also significant at $\mathrm{P} \leq 0.05$ for all traits except Plant height, Number of tillers per hill and leaf drying (Table 3). Under non drought stress environment, 
the genotypes were highly significant at $\mathrm{P} \leq 0.05$ for all the traits. For environment $\times$ genotypes effects, the mean squares were significant at $\mathrm{P} \leq 0.05$ for only chlorophyll content and plant height (Table 4).

Table 3. Analysis of variance for various traits in Under Drought in KALRO Center and Kirogo farm

\begin{tabular}{|c|c|c|c|c|c|c|c|c|c|c|c|c|c|}
\hline \multirow[b]{2}{*}{ Source of Variation } & \multirow[b]{2}{*}{ df } & \multicolumn{3}{|c|}{ Drought Parameters } & \multicolumn{2}{|c|}{ Yield Traits } & \multicolumn{7}{|c|}{ Other Agronomic Traits } \\
\hline & & $\begin{array}{l}\text { LR } \\
(0-9)\end{array}$ & $\begin{array}{l}\text { LD } \\
(0-9)\end{array}$ & $\begin{array}{l}\text { SF } \\
(\%)\end{array}$ & $\begin{array}{l}\text { TGW } \\
(\mathrm{g})\end{array}$ & $\begin{array}{l}\text { GY } \\
(\mathrm{T} / \mathrm{Ha})\end{array}$ & $\begin{array}{l}50 \% \\
\text { DTF }\end{array}$ & $\begin{array}{l}\mathrm{Cc} \\
(\mathrm{Lux})\end{array}$ & $\begin{array}{l}\mathrm{PH} \\
(\mathrm{cm})\end{array}$ & $\mathrm{NTL} / \mathrm{H}$ & $\begin{array}{l}\text { DTM } \\
\text { (Days) }\end{array}$ & $\begin{array}{l}\text { PW } \\
(\mathrm{g})\end{array}$ & $\begin{array}{l}\text { PL } \\
(\mathrm{cm})\end{array}$ \\
\hline Replication & 4 & 1.7 & 0.4 & 209.7 & 23.03 & 2.33 & 23 & 26.3 & 120.8 & 20.46 & 201.44 & 18.9 & 3.7 \\
\hline Environment (E) & 1 & 0.5 & 0.1 & 0.01 & $41.86^{* *}$ & 41.86 & 234 & 0.31 & 0.01 & 0.01 & $31.89^{*}$ & 20.78 & 0.02 \\
\hline Genotype (G) & 35 & $7.3 * * *$ & $0.39 * *$ & $248.4 * * *$ & $100.56^{* *}$ & $3.65 * * *$ & $396^{* * *}$ & $28.0^{* *}$ & $656.1 * * *$ & $30.56 * * *$ & $143.47 * * *$ & $23.80^{* * *}$ & $9.5^{* * *}$ \\
\hline $\mathrm{G}^{*} \mathrm{E}$ & 35 & $1.7 * * *$ & 0.29 & $161.8^{*}$ & $49.78 * * *$ & 2.33 & $61.9^{* *}$ & $26.4 * * *$ & 0.01 & 15.2 & $190.68 * * *$ & $2.96^{* * *}$ & $8.0 * * *$ \\
\hline
\end{tabular}

Note. * Significant at $\mathrm{P} \leq 0.05$, ** Significant at $\mathrm{P} \leq 0.01$ and *** Significant at $\mathrm{P} \leq 0.001, \mathrm{LR}$ - Leaf rolling, LD Leaf drying, SF - Spikelet fertility, TGW - Thousand grain weight, GY- Grain yield, 50\% DTF - 50\% Days to 50\% flowering, Cc - Chlorophyll content, PH - Plant height, NTL/H - Number of tillers per hill, DTM - Day to maturity, PW- Panicle weight, PL - Plant length.

Table 4. Analysis of variance for various traits in Under Non Drought in KALRO Center and Kirogo farm

\begin{tabular}{|c|c|c|c|c|c|c|c|c|c|c|c|}
\hline \multirow{2}{*}{ Source of Variation } & \multirow{2}{*}{$\mathrm{df}$} & \multirow{2}{*}{$\begin{array}{l}\text { Drought Parameter } \\
\text { SF }(\%)\end{array}$} & \multicolumn{2}{|c|}{ Yield Traits } & \multicolumn{7}{|c|}{ Other Agronomic Traits } \\
\hline & & & TGW (g) & GY (T/Ha) & $50 \%$ DTF & $\mathrm{Cc}(\operatorname{Lux})$ & $\mathrm{PH}(\mathrm{cm})$ & $\mathrm{NTL} / \mathrm{H}$ & DTM & PW (g) & PL $(\mathrm{cm})$ \\
\hline Replication & 4 & 341.9 & $37.3^{*}$ & 6.4 & 226.2 & 34.1 & 37.3 & 53.1 & 725.6 & 0.78 & 0.99 \\
\hline Environment (E) & 1 & 0.21 & 0.13 & 1.4 & 416.7 & 186.4 & 0.16 & 0.41 & 0.11 & 0.21 & 0.17 \\
\hline Genotype (G) & 35 & $60.30^{* * *}$ & $113.3^{* * *}$ & $3.70^{* * *}$ & $190.8 * * *$ & $20.1^{* * *}$ & $113^{* *}$ & $11.80 *$ & $251.7 * * *$ & $8.4 * * *$ & $5.00 * *$ \\
\hline $\mathrm{G}^{*} \mathrm{E}$ & 35 & 83.4 & 0.1 & 2.8 & 182.0 & $21.5^{* * *}$ & 0.11 & 9.1 & 0.78 & 0.19 & $4.20 *$ \\
\hline
\end{tabular}

Note. * Significant at $\mathrm{P} \leq 0.05$, ** Significant at $\mathrm{P} \leq 0.01$ and *** Significant at $\mathrm{P} \leq 0.001, \mathrm{SF}-$ Spikelet fertility, TGW - Thousand grain weight, GY - Grain yield, 50\% DTF - 50\% Days to flowering, Cc - Chlorophyll content, PH - Plant height, NTL/H - Number of tillers per Hill, DTM - Day to maturity, PW - Panicle weight, PL - Plant length.

\subsection{Mean Performance for the Six Rice Parents under Non-Drought Conditions}

Parent NERICA $2\left(\mathrm{P}_{2}\right)$ was the highest yielding with $5.2 \mathrm{t} \mathrm{ha}^{-1}$ followed by NERICA $1\left(\mathrm{P}_{1}\right)$ with $5.0 \mathrm{t} \mathrm{ha}^{-1}$ whereas Komboka $\left(\mathrm{P}_{1}\right)$ and Saro $5\left(\mathrm{P}_{5}\right)$ were the lowest grain yielders with $4.0 \mathrm{tha}^{-1}$ and $4.3 \mathrm{t} \mathrm{ha}^{-1}$ respectively. Parent $\mathrm{P}_{2}$ had the highest spikelet fertility (85\%) while; $\mathrm{P}_{1}$ recorded the longest panicle length of $24.9 \mathrm{~cm}$ (Table $5)$.

Table 5. Mean performance for the parents under non drought in KALRO Center and Kirogo research farm

\begin{tabular}{|c|c|c|c|c|c|c|c|c|c|c|c|c|}
\hline \multirow[b]{2}{*}{ Parents } & \multicolumn{3}{|c|}{ Drought Parameters } & \multicolumn{2}{|c|}{ Yield Traits } & \multicolumn{7}{|c|}{ Other Agronomic Traits } \\
\hline & $\begin{array}{l}\text { SF } \\
(\%)\end{array}$ & $\begin{array}{l}\text { LR } \\
(0-9)\end{array}$ & $\begin{array}{l}\text { LD } \\
(0-9)\end{array}$ & $\begin{array}{l}\text { TGW } \\
(\mathrm{g})\end{array}$ & $\begin{array}{l}\text { GY } \\
(\mathrm{T} / \mathrm{Ha})\end{array}$ & $\begin{array}{l}50 \% \text { DTF } \\
\text { (days) }\end{array}$ & $\begin{array}{l}\mathrm{Cc} \\
\text { (Lux) }\end{array}$ & $\begin{array}{l}\text { PL } \\
(\mathrm{cm})\end{array}$ & $\mathrm{NTL} / \mathrm{H}$ & $\begin{array}{l}\text { DTM } \\
\text { (Days) }\end{array}$ & $\begin{array}{l}\text { PW } \\
(\mathrm{g})\end{array}$ & $\begin{array}{l}\mathrm{PH} \\
(\mathrm{cm})\end{array}$ \\
\hline NERICA $1\left(\mathrm{P}_{1}\right)$ & 84 & 1 & 1 & 29.5 & 5.0 & 80 & 47.0 & 24.9 & 14 & 109 & 5.0 & 128 \\
\hline $\operatorname{NERICA} 2\left(\mathrm{P}_{2}\right)$ & 85 & 1 & 1 & 28.3 & 5.2 & 84 & 47.8 & 23.4 & 15 & 110 & 5.7 & 137 \\
\hline NERICA11 $\left(\mathrm{P}_{3}\right)$ & 80 & 1 & 1 & 27.1 & 4.8 & 84 & 42.2 & 21.7 & 14 & 112 & 5.2 & 113 \\
\hline NERICA $15\left(\mathrm{P}_{4}\right)$ & 83 & 1 & 1 & 29.7 & 4.8 & 83 & 46.5 & 24.2 & 15 & 113 & 5.6 & 140 \\
\hline SARO $5\left(\mathrm{P}_{5}\right)$ & 78 & 1 & 1 & 19.6 & 4.3 & 80 & 42.5 & 23.2 & 17 & 128 & 4.5 & 86 \\
\hline Komboka $\left(\mathrm{P}_{6}\right)$ & 79 & 1 & 1 & 20.7 & 4.0 & 72 & 44.7 & 23.3 & 16 & 134 & 2.7 & 105 \\
\hline Grand Means & 81 & 1 & 1 & 26 & 4.6 & 90 & 45 & 23 & 15 & 118 & 5 & 118 \\
\hline
\end{tabular}

Note. SF - Spikelet fertility, LR - Leaf rolling, LD - Leaf drying, TGW - Thousand grain weight, GY - Grain yield; DTF - 50\% Days to flowering, Cc - Chlorophyll content, PL - Panicle length, NTL/H - Number of tillers per hill, DTM - Day to maturity, PW - Panicle weight, PH - Plant height. 


\subsection{Mean Performance of the Generated Crosses under Non-Drought Conditions}

The highest grain yielder crosses under non-drought condition were $\mathrm{P}_{2} \times \mathrm{P}_{4}, \mathrm{P}_{1} \times \mathrm{P}_{2}$ and $\mathrm{P}_{2} \times \mathrm{P}_{5}$ with 5.6, 5.5 and $5.4 \mathrm{t} \mathrm{ha}^{-1}$ respectively. Moreover, these crosses had the highest number of reproductive tillers of 18,16 and 17 tillers/hill respectively. The least in grain yield was $\mathrm{P}_{1} \times \mathrm{P}_{3}$ and $\mathrm{P}_{2} \times \mathrm{P}_{6}$ with $3.6 \mathrm{tha}^{-1}$ and $4.0 \mathrm{t} \mathrm{ha}^{-1}$. Besides, low grain yield both crosses had the least spikelet fertility of $79 \%$ (Table 6).

Table 6. Mean performance for the generated crosses under non drought in KALRO Center and Kirogo research farm

\begin{tabular}{|c|c|c|c|c|c|c|c|c|c|c|c|c|}
\hline \multirow[b]{2}{*}{ Crosses } & \multicolumn{3}{|c|}{ Drought Parameters } & \multicolumn{2}{|c|}{ Yield Traits } & \multicolumn{7}{|c|}{ Other Agronomic Traits } \\
\hline & $\begin{array}{l}\text { SF } \\
(\%)\end{array}$ & $\begin{array}{l}\text { LR } \\
(0-9)\end{array}$ & $\begin{array}{l}\text { LD } \\
(0-9)\end{array}$ & $\begin{array}{l}\text { TGW } \\
(\mathrm{g})\end{array}$ & $\begin{array}{l}\text { GY } \\
(\mathrm{T} / \mathrm{Ha})\end{array}$ & $\begin{array}{l}50 \% \text { DTF } \\
\text { (days) }\end{array}$ & $\begin{array}{l}\mathrm{Cc} \\
\text { (Lux) }\end{array}$ & $\begin{array}{l}\text { PL } \\
(\mathrm{cm})\end{array}$ & $\mathrm{NTL} / \mathrm{H}$ & $\begin{array}{l}\text { DTM } \\
\text { (Days) }\end{array}$ & $\begin{array}{l}\text { PW } \\
\text { (g) }\end{array}$ & $\begin{array}{l}\mathrm{PH} \\
(\mathrm{cm})\end{array}$ \\
\hline $\mathrm{P}_{1} \times \mathrm{P}_{2}$ & 84 & 1 & 1 & 27.7 & 5.5 & 85 & 47.0 & 24.8 & 16 & 121 & 5.0 & 120 \\
\hline $\mathrm{P}_{1} \times \mathrm{P}_{3}$ & 79 & 1 & 1 & 31.5 & 3.6 & 82 & 44.4 & 24.9 & 13 & 113 & 5.3 & 125 \\
\hline $\mathrm{P}_{1} \times \mathrm{P}_{4}$ & 86 & 1 & 1 & 28.7 & 4.4 & 82 & 47.4 & 23.7 & 15 & 113 & 5.2 & 123 \\
\hline $\mathrm{P}_{1} \times \mathrm{P}_{5}$ & 83 & 1 & 1 & 30.5 & 5.3 & 83 & 48.5 & 23.8 & 14 & 112 & 5.2 & 115 \\
\hline $\mathrm{P}_{1} \times \mathrm{P}_{6}$ & 88 & 1 & 1 & 29.2 & 5.0 & 85 & 46.8 & 25.7 & 13 & 108 & 4.8 & 118 \\
\hline $\mathrm{P}_{2} \times \mathrm{P}_{3}$ & 83 & 1 & 1 & 26.5 & 4.0 & 83 & 47.4 & 25.8 & 14 & 109 & 5.7 & 132 \\
\hline $\mathrm{P}_{2} \times \mathrm{P}_{4}$ & 81 & 1 & 1 & 20.5 & 5.6 & 83 & 45.0 & 22.5 & 18 & 112 & 3.9 & 124 \\
\hline $\mathrm{P}_{2} \times \mathrm{P}_{5}$ & 80 & 1 & 1 & 18.9 & 5.4 & 83 & 42.8 & 22.8 & 13 & 108 & 4.9 & 112 \\
\hline $\mathrm{P}_{2} \times \mathrm{P}_{6}$ & 82 & 1 & 1 & 37.7 & 4.1 & 87 & 47.4 & 23.1 & 17 & 116 & 5.8 & 132 \\
\hline $\mathrm{P}_{3} \times \mathrm{P}_{4}$ & 83 & 1 & 1 & 30.3 & 4.7 & 84 & 47.5 & 24.5 & 15 & 115 & 5.6 & 132 \\
\hline $\mathrm{P}_{3} \times \mathrm{P}_{6}$ & 85 & 1 & 1 & 32.6 & 5.2 & 104 & 47.0 & 24.3 & 15 & 128 & 5.4 & 129 \\
\hline $\mathrm{P}_{3} \times \mathrm{P}_{6}$ & 83 & 1 & 1 & 27.4 & 5.1 & 88 & 48.4 & 24.0 & 15 & 120 & 5.0 & 127 \\
\hline $\mathrm{P}_{4} \times \mathrm{P}_{5}$ & 84 & 1 & 1 & 32.8 & 5.2 & 87 & 48.0 & 23.7 & 13 & 125 & 5.5 & 124 \\
\hline $\mathrm{P}_{5} \times \mathrm{P}_{6}$ & 84 & 1 & 1 & 29.4 & 5.3 & 95 & 47.9 & 24.7 & 14 & 110 & 4.8 & 117 \\
\hline $\mathrm{P}_{4} \times \mathrm{P}_{6}$ & 79 & 1 & 1 & 33.1 & 4.0 & 98 & 46.2 & 24.1 & 16 & 130 & 5.5 & 124 \\
\hline Grand Means & 83 & 1 & 1 & 29 & 4.8 & 87 & 47 & 24 & 15 & 116 & 5.0 & 124 \\
\hline Overall Means & 84 & 1 & 1 & 28 & 4.7 & 90 & 46 & 24 & 15 & 118 & 5.0 & 122 \\
\hline $\operatorname{LSD}(5 \%)$ & 6.5 & 0 & 0 & 12.3 & 1.2 & 8.3 & 6.7 & 5.1 & 2.7 & 6.5 & 2.9 & 8.1 \\
\hline CV $(\%)$ & 15.1 & 0 & 0 & 4.1 & 1.1 & 8.5 & 3.5 & 1.4 & 3.9 & 12.5 & 0.5 & 11.4 \\
\hline
\end{tabular}

Note. $\mathrm{P}_{1}$ - NERICA 1, $\mathrm{P}_{2}$ - NERICA 2, $\mathrm{P}_{3}$ - NERICA 11, $\mathrm{P}_{4}$ - NERICA 15, $\mathrm{P}_{5}-\mathrm{SARO5}, \mathrm{P}_{6}$ - Komboka, SF Spikelet fertility, LR - Leaf rolling, LD - Leaf drying, TGW - Thousand grain weight, GY - Grain yield; DTF $50 \%$ Days to flowering, Cc - Chlorophyll content, PL - Panicle Length, NTL/H - Number of tillers per hill, DTM - Day to maturity, PW - Panicle weight, PH - Plant Height.

\subsection{Mean Performance of the Six Rice Parents Crosses under Drought Conditions}

Based on drought tolerance parameters, NERICA $15\left(\mathrm{P}_{4}\right)$ had high spikelet fertility with $81 \%$ followed by NERICA1 $\left(\mathrm{P}_{1}\right)$ with $75 \%$ and NERICA2 $\left(\mathrm{P}_{2}\right)$ with $74 \%$. Parent NERICA $15\left(\mathrm{P}_{4}\right)$ and NERICA $2\left(\mathrm{P}_{2}\right)$ exhibited low scores of leaf drying and leaf rolling both with scores of 2 . For grain yield parent NERICA 15 (P4) was the highest with $4.2 \mathrm{t} \mathrm{ha}^{-1}$ followed by NERICA 11 with $3.7 \mathrm{t} \mathrm{ha}^{-1}$. On other hand SARO $5\left(\mathrm{P}_{5}\right)$ and Komboka $\left(\mathrm{P}_{6}\right)$ recorded low grain yield of 2.0 and $2.1 \mathrm{tha}^{-1}$. Besides this low yield the two parents had lowest spikelet fertility and highest scores (5) of leaf rolling (Table 7). 
Table 7. Mean performance for the parents under drought in KALRO Center and Kirogo research farm

\begin{tabular}{|c|c|c|c|c|c|c|c|c|c|c|c|c|}
\hline \multirow[b]{2}{*}{ Parents } & \multicolumn{3}{|c|}{ Drought Parameters } & \multicolumn{2}{|c|}{ Yield Traits } & \multicolumn{7}{|c|}{ Other Agronomic Traits } \\
\hline & $\begin{array}{l}\text { SF } \\
(\%)\end{array}$ & $\begin{array}{l}\text { LR } \\
(0-9)\end{array}$ & $\begin{array}{l}\text { LD } \\
(0-9)\end{array}$ & $\begin{array}{l}\text { TGW } \\
(\mathrm{g})\end{array}$ & $\begin{array}{l}\text { GY } \\
(\mathrm{T} / \mathrm{Ha})\end{array}$ & $\begin{array}{l}50 \mathrm{DTF} \\
\text { (days) }\end{array}$ & $\begin{array}{l}\mathrm{Cc} \\
(\operatorname{Lux})\end{array}$ & $\begin{array}{l}\mathrm{PL} \\
(\mathrm{cm})\end{array}$ & $\mathrm{NTL} / \mathrm{H}$ & $\begin{array}{l}\text { DTM } \\
\text { (Days) }\end{array}$ & $\begin{array}{l}\text { PW } \\
(\mathrm{g})\end{array}$ & $\begin{array}{l}\mathrm{PH} \\
(\mathrm{cm})\end{array}$ \\
\hline NERICA $1\left(\mathrm{P}_{1}\right)$ & 75 & 4 & 2 & 28.7 & 3.5 & 84 & 47.8 & 20.3 & 9 & 108 & 4.8 & 112 \\
\hline NERICA $2\left(\mathrm{P}_{2}\right)$ & 74 & 2 & 2 & 24.6 & 3.7 & 85 & 46.7 & 24.8 & 9 & 113 & 4.6 & 105 \\
\hline NERICA11 $\left(\mathrm{P}_{3}\right)$ & 73 & 4 & 2 & 24.0 & 3.4 & 86 & 45.5 & 21.8 & 8 & 111 & 4.6 & 99 \\
\hline NERICA $15\left(\mathrm{P}_{4}\right)$ & 81 & 2 & 2 & 30.0 & 4.2 & 87 & 46.6 & 24.3 & 9 & 111 & 4.7 & 133 \\
\hline SARO $5\left(\mathrm{P}_{5}\right)$ & 61 & 5 & 3 & 18.6 & 2.0 & 101 & 42.5 & 19.9 & 13 & 129 & 3.1 & 84 \\
\hline $\operatorname{Komboka}\left(\mathrm{P}_{6}\right)$ & 72 & 5 & 2 & 20.0 & 2.1 & 102 & 42.0 & 22.5 & 11 & 132 & 2.0 & 107 \\
\hline Grand Means & 73 & 4 & 2 & 24 & 3.1 & 91 & 45 & 22 & 10 & 117 & 4 & 107 \\
\hline
\end{tabular}

Note. SF - Spikelet fertility, LR - Leaf rolling, LD - Leaf drying, TGW - Thousand grain weight, GY - Grain yield, DTF - 50\% Days to flowering, Cc - Chlorophyll content, PL - Panicle length, NTL/H - Number of tillers per hill, DTM - Day to maturity, PW - Panicle weight, PH - Plant height.

\subsection{Mean Performance for the Generated Crosses under Drought Conditions}

Hybrid crosses of $\mathrm{P}_{3} \times \mathrm{P}_{4}, \mathrm{P}_{2} \times \mathrm{P}_{4}$ and $\mathrm{P}_{2} \times \mathrm{P}_{3}$ exhibited high spikelet fertility and grain yield with $86,83,82 \%$ and $81 \%$ and a grain yield of $4.8,5.2$ and $4.6 \mathrm{t} \mathrm{ha}^{-1}$ respectively. Moreover, all these hybrids manifested low scores of leaf drying and rolling ranging between 2 and 3 . The poor grain yielders were crosses such as $\mathrm{P}_{6} \times \mathrm{P}_{4}$, $\mathrm{P}_{5} \times \mathrm{P}_{3}$ and $\mathrm{P}_{5} \times \mathrm{P}_{1}$ with 2.2, 2.0 and $3.0 \mathrm{t} \mathrm{ha}^{-1}$ respectively. The same crosses recorded low spikelet fertility of less than $70 \%$ and high morphological scores such as leaf rolling of 5 in all crosses (Table 8 ).

Table 8. Mean performance of the generated crosses under drought in KALRO Center and Kirogo research farm

\begin{tabular}{|c|c|c|c|c|c|c|c|c|c|c|c|c|}
\hline \multirow[b]{2}{*}{ Crosses } & \multicolumn{3}{|c|}{ Drought Parameters } & \multicolumn{2}{|c|}{ Yield Traits } & \multicolumn{7}{|c|}{ Other Agronomic Traits } \\
\hline & SF (\%) & $\begin{array}{l}\text { LR } \\
(0-9)\end{array}$ & $\begin{array}{l}\text { LD } \\
(0-9)\end{array}$ & $\begin{array}{l}\text { TGW } \\
(\mathrm{g})\end{array}$ & $\begin{array}{l}\text { GY } \\
(\mathrm{T} / \mathrm{Ha})\end{array}$ & $\begin{array}{l}50 \% \text { DTF } \\
\text { (days) }\end{array}$ & $\begin{array}{l}\text { Cc } \\
\text { (Lux) }\end{array}$ & $\begin{array}{l}\mathrm{PL} \\
(\mathrm{cm})\end{array}$ & $\mathrm{NTL} / \mathrm{H}$ & $\begin{array}{l}\text { DTM } \\
\text { (Days) }\end{array}$ & $\begin{array}{l}\text { PW } \\
\text { (g) }\end{array}$ & $\begin{array}{l}\mathrm{PH} \\
(\mathrm{cm})\end{array}$ \\
\hline $\mathrm{P}_{1} \times \mathrm{P}_{2}$ & 68 & 3 & 3 & 28.8 & 4.2 & 87 & 44.5 & 22.4 & 10 & 110 & 4.3 & 102 \\
\hline $\mathrm{P}_{1} \times \mathrm{P}_{3}$ & 76 & 3 & 2 & 29.9 & 4.0 & 85 & 47.8 & 23.2 & 9 & 107 & 3.8 & 105 \\
\hline $\mathrm{P}_{1} \times \mathrm{P}_{4}$ & 77 & 3 & 2 & 24.2 & 4.5 & 90 & 46.8 & 22.4 & 9 & 111 & 3.9 & 103 \\
\hline $\mathrm{P}_{1} \times \mathrm{P}_{5}$ & 78 & 3 & 2 & 24.3 & 3.8 & 85 & 45.5 & 23.6 & 8 & 118 & 4.2 & 101 \\
\hline $\mathrm{P}_{1} \times \mathrm{P}_{6}$ & 78 & 4 & 2 & 28.9 & 4.2 & 88 & 46.5 & 21.8 & 11 & 117 & 4.7 & 107 \\
\hline $\mathrm{P}_{2} \times \mathrm{P}_{3}$ & 82 & 2 & 2 & 25.4 & 4.6 & 82 & 48.0 & 21.4 & 12 & 114 & 4.7 & 109 \\
\hline $\mathrm{P}_{2} \times \mathrm{P}_{4}$ & 83 & 2 & 2 & 22.6 & 5.2 & 84 & 46.7 & 23.7 & 13 & 116 & 3.8 & 104 \\
\hline $\mathrm{P}_{2} \times \mathrm{P}_{5}$ & 64 & 3 & 2 & 26.7 & 3.8 & 88 & 46.2 & 23.2 & 8 & 119 & 4.5 & 108 \\
\hline $\mathrm{P}_{2} \times \mathrm{P}_{6}$ & 70 & 3 & 2 & 20.9 & 4.5 & 85 & 47.0 & 22.3 & 9 & 115 & 5.3 & 123 \\
\hline $\mathrm{P}_{3} \times \mathrm{P}_{4}$ & 86 & 3 & 2 & 30.7 & 4.8 & 88 & 46.3 & 21.5 & 11 & 113 & 4.3 & 115 \\
\hline $\mathrm{P}_{3} \times \mathrm{P}_{5}$ & 69 & 4 & 3 & 20.5 & 4.4 & 106 & 43.4 & 19.4 & 12 & 127 & 2.8 & 98 \\
\hline $\mathrm{P}_{3} \times \mathrm{P}_{6}$ & 68 & 4 & 2 & 26.1 & 4.3 & 91 & 48.6 & 22.2 & 7 & 115 & 4.3 & 108 \\
\hline $\mathrm{P}_{4} \times \mathrm{P}_{5}$ & 66 & 4 & 2 & 29.3 & 3.8 & 92 & 46.9 & 23.4 & 8 & 117 & 4.9 & 110 \\
\hline $\mathrm{P}_{5} \times \mathrm{P}_{6}$ & 70 & 5 & 2 & 29.6 & 4.5 & 97 & 49.1 & 23.0 & 10 & 116 & 4.8 & 122 \\
\hline $\mathrm{P}_{4} \times \mathrm{P}_{6}$ & 76 & 6 & 3 & 17.7 & 4.4 & 96 & 41.4 & 20.3 & 15 & 132 & 2.5 & 88 \\
\hline Grand Means & 73 & 3 & 2 & 26 & 4.3 & 90 & 46 & 22 & 10 & 116 & 4 & 107 \\
\hline $\operatorname{LSD}(5 \%)$ & 18 & 1.7 & 0.8 & 4.3 & 2.2 & 4.2 & 5.1 & 5.3 & 2.1 & 3.5 & 2.6 & 10 \\
\hline CV (\%) & 15 & 1 & 0.4 & 3.8 & 1.05 & 4.4 & 2.6 & 1.4 & 2.7 & 4.6 & 1.4 & 12 \\
\hline
\end{tabular}

Note. $\mathrm{P}_{1}$ - NERICA 1, $\mathrm{P}_{2}$ - NERICA 2, $\mathrm{P}_{3}$-NERICA 11; $\mathrm{P}_{4}$ - NERICA 15, $\mathrm{P}_{5}$ - SARO5, $\mathrm{P}_{6}$ - Komboka, SF Spikelet Fertility, LR - Leaf rolling, LD - Leaf drying, TGW - Thousand grain weight, GY - Grain yield; DTF - 50\% Days to flowering, Cc - Chlorophyll content, PL - Panicle length, NTL/H - Number of tillers per hill, DTM - Day to maturity, PW - Panicle weight, PH - Plant height. 


\subsection{Analysis of Variance for Combining Abilities for Various Traits}

\subsubsection{Analysis of Variance for Combining Abilities for Various Traits under Drought Conditions}

Analysis of variance of combining ability showed that the mean square of the GCA, SCA and RCA were significant at $\mathrm{P} \leq 0.05$ for all drought tolerance traits, yield components and agronomic traits evaluated except days to maturity (Table 9). The variance for GCA includes the additive portion of the total whereas the variance for SCA comprises the non additive portion of the total variance as a result of dominance and epistasis. Non-additive gene action was predominant for spikelet fertility, leaf rolling, leaf drying, grain yield, thousand grain weights, chlorophyll content and number of tillers per hill. Additive gene action was predominant for days to $50 \%$ flowering, and panicle length. Plant height and days to maturity were governed by both additive and non additive gene actions (Table 9).

\subsubsection{Analysis of Variance for Combining Abilities for Various Traits under Non-Drought Conditions}

The mean squares of GCA, SCA and RCA were significant at $\mathrm{P} \leq 0.05$ for all traits except spikelet fertility, chlorophyll content and days to maturity. Additive gene action was predominant for days to $50 \%$ flowering, panicle length and days to maturity while panicle weight and chlorophyll were governed by both additive and non additive gene actions (Table 10).

Table 9. Analysis of variance for combining abilities for various traits under drought in KALRO Center and Kirogo research farm

\begin{tabular}{|c|c|c|c|c|c|c|c|c|c|c|c|c|c|}
\hline \multirow[b]{2}{*}{ Source of Variation } & \multirow[b]{2}{*}{ df } & \multicolumn{3}{|c|}{ Drought Parameters } & \multicolumn{2}{|c|}{ Yield Traits } & \multicolumn{7}{|c|}{ Other Agronomic Traits } \\
\hline & & $\begin{array}{l}\text { SF } \\
(\%)\end{array}$ & $\begin{array}{l}\text { LR } \\
(0-9)\end{array}$ & $\begin{array}{l}\text { LD } \\
(0-9)\end{array}$ & $\begin{array}{l}\text { TGW } \\
\text { (g) }\end{array}$ & $\begin{array}{l}\text { GY } \\
(\mathrm{T} / \mathrm{Ha})\end{array}$ & $\begin{array}{l}50 \% \text { DTF } \\
\text { (Days) }\end{array}$ & $\begin{array}{l}\mathrm{Cc} \\
(\operatorname{Lux})\end{array}$ & $\begin{array}{l}\text { PL } \\
(\mathrm{cm})\end{array}$ & $\mathrm{NTL} / \mathrm{H}$ & $\begin{array}{l}\text { DTM } \\
\text { (Days) }\end{array}$ & $\begin{array}{l}\text { PW } \\
(\mathrm{g})\end{array}$ & $\mathrm{PH}(\mathrm{cm})$ \\
\hline GCA & 5 & $640.8^{* *}$ & $18.9^{* *}$ & $0.54 * *$ & $98.8 * *$ & $78.57 * *$ & $1527 * *$ & $32.4 * *$ & $9.3^{* *}$ & $40.8 * *$ & 1956.5 & $98.8^{* *}$ & $577.9^{*}$ \\
\hline SCA & 15 & $229.5^{* *}$ & $8.46^{* *}$ & $0.32 * *$ & $169.2^{* *}$ & $113.3^{* *}$ & $181 * *$ & $37.8 * *$ & $10.2 * *$ & $25.3 * *$ & 131.1 & $169.2 * *$ & $1180^{*}$ \\
\hline $\mathrm{RCA}$ & 15 & $139.6^{* *}$ & $2.8 * *$ & $0.40 * *$ & $32.5 * *$ & $69.5 * *$ & $251 * *$ & $16.8 * *$ & $4.4 * *$ & $13.8 * *$ & 188.8 & $32.49 * *$ & $158^{*}$ \\
\hline$\sigma_{\mathrm{A}}^{2}$ & & 3.9 & 0.01 & 0.003 & 0.25 & 0.1 & 0.68 & 0.5 & 0.26 & 0.17 & 3.01 & 0.01 & 0.001 \\
\hline$\sigma^{2}{ }_{D}$ & & 10.75 & 0.14 & 0.02 & 0.68 & 0.32 & 0.9 & 1.9 & 0.48 & 1.95 & 6.1 & 0.06 & 0.002 \\
\hline$\sigma_{A}^{2} / \sigma_{D}^{2}$ & & 0.4 & 0.1 & 0.2 & 0.4 & 0.38 & 0.6 & 0.4 & 0.6 & 0.1 & 0.5 & 0.3 & 0.5 \\
\hline
\end{tabular}

Note. ${ }^{*}$ Significant at $\mathrm{P} \leq 0.05, * *$ Significant at $\mathrm{P} \leq 0.01$ and $* *$ Significant at $\mathrm{P} \leq 0.001, \sigma_{\mathrm{A}}{ }_{\mathrm{A}}$ - Additive Variance, $\sigma_{D}^{2}$ - Dominance variance, GCA - General combining ability, SCA - Specific combining ability, RCA Reciprocal combining ability, SF - Spikelet fertility, LR - Leaf rolling, LD - Leaf drying, TGW - Thousand grain weight, GY Grain yield, DTF - 50\% Days to flowering, Cc - Chlorophyll content, PL - Panicle Length, NTL/H Number of tillers per hill, DTM - Day to maturity, PW - Panicle weight, PH - Plant height.

Table 10. Analysis of variance for combining abilities for various traits under non drought in KALRO Center and Kirogo research farm

\begin{tabular}{|c|c|c|c|c|c|c|c|c|c|c|c|}
\hline \multirow[b]{2}{*}{ Source of Variation } & \multirow[b]{2}{*}{$\mathrm{df}$} & \multirow{2}{*}{$\begin{array}{l}\text { Drought Parameter } \\
\text { SF }(\%)\end{array}$} & \multicolumn{2}{|c|}{ Yield Traits } & \multicolumn{7}{|c|}{ Other Agronomic Traits } \\
\hline & & & $\begin{array}{l}\text { TGW } \\
(\mathrm{g})\end{array}$ & $\begin{array}{l}\text { GY } \\
(\mathrm{T} / \mathrm{Ha})\end{array}$ & $\begin{array}{l}50 \% \text { DTF } \\
\text { (Days) }\end{array}$ & $\begin{array}{l}\mathrm{Cc} \\
\text { (Lux) }\end{array}$ & $\begin{array}{l}\mathrm{PL} \\
(\mathrm{cm})\end{array}$ & $\mathrm{NTL} / \mathrm{H}$ & $\begin{array}{l}\text { DTM } \\
\text { (Days) }\end{array}$ & $\begin{array}{l}\text { PW } \\
(\mathrm{g})\end{array}$ & $\begin{array}{l}\mathrm{PH} \\
(\mathrm{cm})\end{array}$ \\
\hline GCA & 5 & 20.2 & $89.1 * *$ & $7.1^{* *}$ & $412.6^{* *}$ & 37.1 & $10.7 * *$ & $15.2 * *$ & 406.9 & $10.2 * *$ & $89.1^{*}$ \\
\hline SCA & 15 & 49.9 & $115.1 * *$ & $1.5^{* *}$ & $142.6^{* *}$ & 17.5 & $4.6^{* *}$ & $10.1 * *$ & 350.3 & $10.7 * *$ & $115.1^{*}$ \\
\hline RCA & 15 & 96.2 & $119.6^{* *}$ & $3.4^{* *}$ & $92.8 * *$ & 16.9 & $4.3^{* *}$ & $6.25 * *$ & 101.3 & $5.4^{* *}$ & $119.6^{*}$ \\
\hline$\sigma_{\mathrm{A}}^{2}$ & & 0.54 & 0.24 & 0.02 & 6.7 & 0.5 & 0.07 & 0.08 & 3.01 & 0.02 & 0.46 \\
\hline$\sigma_{D}^{2}$ & & 3.3 & 1.8 & 0.19 & 5.7 & 0.9 & 0.03 & 0.61 & 4.8 & 0.04 & 14.24 \\
\hline$\sigma_{\mathrm{A}}^{2} / \sigma_{\mathrm{D}}^{2}$ & & 0.2 & 0.2 & 0.2 & 0.7 & 0.5 & 0.8 & 0.2 & 0.6 & 0.5 & 0.1 \\
\hline
\end{tabular}

Note. * Significant at $\mathrm{P} \leq 0.05$, ** Significant at $\mathrm{P} \leq 0.01$ and $* * *$ Significant at $\mathrm{P} \leq 0.001, \sigma_{\mathrm{A}}{ }^{2}$ - Additive Variance, $\sigma_{D}^{2}$ - Dominance variance, GCA - General combining ability, SCA - Specific combining ability, RCA Reciprocal combining ability, SF - Spikelet fertility, TGW - Thousand grain weight, GY - Grain yield, DTF $50 \%$ Days to flowering, Cc - Chlorophyll content, PL - Panicle Length, NTL/H - Number of tillers per hill, DTM - Day to maturity, PW - Panicle weight, PH - Plant Height. 


\subsection{Combining Ability Estimates}

\subsubsection{General Combining Ability (GCA) Effects}

The parent NERICA $2\left(\mathrm{P}_{2}\right)$, NERICA $1\left(\mathrm{P}_{1}\right)$ and NERICA $15\left(\mathrm{P}_{5}\right)$ had highest positive GCA effects for spikelet fertility of 3.41, 2.76 and 2.64 respectively (Table 11). Based on leaf rolling and leaf drying score NERICA $2\left(\mathrm{P}_{2}\right)$ and NERICA $15\left(\mathrm{P}_{5}\right)$ had the highest negative GCA effects of -0.78 and -0.07 for leaf rolling and -0.02 and -0.07 for leaf drying respectively. The highest grain yielder was NERICA $15\left(\mathrm{P}_{4}\right)$, NERICA $2\left(\mathrm{P}_{2}\right)$ and NERICA11 $\left(\mathrm{P}_{3}\right)$ with positive GCA effects of $0.8,0.53$ and 0.25 respectively. Besides, the two parents had minimum days to $50 \%$ flowering. In contrast, SARO $5\left(\mathrm{P}_{5}\right)$ and Komboka $\left(\mathrm{P}_{6}\right)$ had low GCA effect for spikelet fertility of 0.06 and -3.86 and positive GCA for leaf rolling and drying. Moreover, these two parents had negative GCA effects on grain yield in spite of showing positive GCA effect for number of tillers per hill of 1.18 and 1.14 respectively (Table 11).

Table 11. General combining ability (GCA) effects for observed traits under drought environment in KALRO Center and Kirogo research farm

\begin{tabular}{|c|c|c|c|c|c|c|c|c|c|c|c|c|}
\hline \multirow[b]{2}{*}{ Parents } & \multicolumn{3}{|c|}{ Drought Parameters } & \multicolumn{2}{|c|}{ Yield Traits } & \multicolumn{7}{|c|}{ Other Agronomic Traits } \\
\hline & $\begin{array}{l}\text { SF } \\
(\%)\end{array}$ & $\begin{array}{l}\text { LR } \\
(0-9)\end{array}$ & $\begin{array}{l}\mathrm{LD} \\
(0-9)\end{array}$ & $\begin{array}{l}\text { TGW } \\
\text { (g) }\end{array}$ & $\begin{array}{l}\text { GY } \\
\text { (T/Ha) }\end{array}$ & $\begin{array}{l}50 \% \text { DTF } \\
\text { (days) }\end{array}$ & $\begin{array}{l}\mathrm{Cc} \\
\text { (Lux) }\end{array}$ & $\begin{array}{l}\mathrm{PL} \\
(\mathrm{cm})\end{array}$ & $\mathrm{NTL} / \mathrm{H}$ & $\begin{array}{l}\text { DTM } \\
\text { (Days) }\end{array}$ & $\begin{array}{l}\mathrm{PW} \\
(\mathrm{g})\end{array}$ & $\begin{array}{l}\mathrm{PH} \\
(\mathrm{cm})\end{array}$ \\
\hline NERICA $1\left(\mathrm{P}_{1}\right)$ & $2.76^{* * *}$ & -0.23 & 0.08 & $1.03 * * *$ & 0.04 & $-4.13 * * *$ & $0.74 * * *$ & 0.22 & $-0.93 *$ & $-4.28 * * *$ & $0.71^{* * *}$ & -1.58 \\
\hline NERICA $2\left(\mathrm{P}_{2}\right)$ & $3.41 * *$ & $-0.78^{* * *}$ & $-0.02 * * *$ & -0.1 & $0.53 * * *$ & $-4.13 * * *$ & $0.62 * * *$ & $0.49 *$ & -0.57 & $-4.67 * * *$ & $0.45^{* * *}$ & $2.84 * *$ \\
\hline NERICA $11\left(\mathrm{P}_{3}\right)$ & $1.82^{* * *}$ & 0.3 & -0.03 & 0.28 & 0.25 & -5.54 & 0.12 & -3.67 & 0.42 & $-2.67 * * *$ & -0.03 & 0.04 \\
\hline NERICA $15\left(\mathrm{P}_{4}\right)$ & $2.64 * * *$ & $-0.27 * * *$ & $-0.07 * * *$ & $1.32 * * *$ & $0.80^{* * *}$ & $-3.15^{* * *}$ & 2.09 & 0.18 & -0.23 & -4.7 & $0.52^{* * *}$ & $3.30^{* * *}$ \\
\hline SARO $5\left(\mathrm{P}_{5}\right)$ & 0.06 & 0.56 & 0.15 & $-1.86^{* * *}$ & $-0.33^{* * *}$ & $7.14 * * *$ & -0.97 & -0.42 & $1.18^{* * *}$ & 7.2 & 0.24 & -0.3 \\
\hline Komboka $\left(\mathrm{P}_{6}\right)$ & -3.86 & $0.41 * * *$ & 0.02 & $-0.66 * * *$ & -0.18 & $4.06^{* * *}$ & -0.59 & -0.11 & $1.14 * * *$ & $5.86^{* * *}$ & -0.17 & -4.3 \\
\hline
\end{tabular}

Note. * Significant at $\mathrm{P} \leq 0.05, * *$ Significant at $\mathrm{P} \leq 0.01$ and $* * *$ Significant at $\mathrm{P} \leq 0.001, \mathrm{P}_{1}-$ NERICA $1, \mathrm{P}_{2}$ NERICA 2, $\mathrm{P}_{3}$ - NERICA 11; $\mathrm{P}_{4}$ - NERICA 15, $\mathrm{P}_{5}-\mathrm{SARO5,} \mathrm{P}_{6}$ - Komboka, SF - Spikelet Fertility, LR - Leaf rolling, LD - Leaf drying, TGW - Thousand grain weight, GY - Grain yield, DTF - 50\% Days to flowering, Cc Chlorophyll content, PL - Panicle Length, NTL/H - Number of tillers per hill, DTM - Day to maturity, PW Panicle weight, PH - Plant Height.

\subsubsection{Specific Combining Ability (SCA) Effects}

The specific combining ability was done for only crosses since, most of reciprocal crosses showed no significant effect for most of the traits evaluated. The hybrid $\mathrm{P}_{2} \times \mathrm{P}_{4}, \mathrm{P}_{1} \times \mathrm{P}_{4}$ and $\mathrm{P}_{2} \times \mathrm{P}_{3}$ expressed high positive SCA effect for spikelet fertility of 5.37, 5.06 and 4.40 respectively. Moreover, $\mathrm{P}_{1} \times \mathrm{P}_{4}, \mathrm{P}_{3} \times \mathrm{P}_{4}$ and $\mathrm{P}_{1} \times \mathrm{P}_{3}$ had low negative SCA effects for leaf rolling score. The best specific combiner for grain yield were $\mathrm{P}_{2} \times \mathrm{P}_{4}, \mathrm{P}_{2} \times \mathrm{P}_{6}, \mathrm{P}_{3} \times \mathrm{P}_{4}$, with a positive SCA effects of 2.71, 1.27 and 1.19 respectively. Hybrid cross of $\mathrm{P}_{3} \times \mathrm{P}_{4}$ had also good SCA effects for panicle weight while a cross of $\mathrm{P}_{2} \times \mathrm{P}_{4}$, and $\mathrm{P}_{1} \times \mathrm{P}_{4}$ exhibited good SCA for minimum days to $50 \%$ flowering (Table 12). $\mathrm{P}_{1} \times \mathrm{P}_{4}, \mathrm{P}_{3} \times \mathrm{P}_{4}$ and $\mathrm{P}_{2} \times \mathrm{P}_{4}$ were overall best crosses for most traits evaluated. These crosses had positive value for leaf rolling in addition to thousand grain weight and $50 \%$ days to flowering (Table 12 ). 
Table 12. Specific combining ability (SCA) effects for observed traits under drought environment in KALRO Center and Kirogo research farm

\begin{tabular}{|c|c|c|c|c|c|c|c|c|c|c|c|c|}
\hline \multirow[b]{2}{*}{ Crosses } & \multicolumn{3}{|c|}{ Drought Parameters } & \multicolumn{2}{|c|}{ Yield Traits } & \multicolumn{7}{|c|}{ Other Agronomic Traits } \\
\hline & $\begin{array}{l}\text { SF } \\
(\%)\end{array}$ & $\begin{array}{l}\text { LR } \\
(0-9)\end{array}$ & $\begin{array}{l}\text { LD } \\
(0-9)\end{array}$ & $\begin{array}{l}\text { TGW } \\
(\mathrm{g})\end{array}$ & $\begin{array}{l}\text { GY } \\
(\mathrm{T} / \mathrm{Ha})\end{array}$ & $\begin{array}{l}50 \% \text { DTF } \\
\text { (days) }\end{array}$ & $\begin{array}{l}\mathrm{Cc} \\
\text { (Lux) }\end{array}$ & $\begin{array}{l}\mathrm{PL} \\
(\mathrm{cm})\end{array}$ & NTL/H & $\begin{array}{l}\text { DTM } \\
\text { (Days) }\end{array}$ & $\begin{array}{l}\text { PW } \\
(\mathrm{g})\end{array}$ & $\begin{array}{l}\mathrm{PH} \\
(\mathrm{cm})\end{array}$ \\
\hline $\mathrm{P}_{1} \times \mathrm{P}_{2}$ & -3.04 & 0.38 & $-0.18 * * *$ & -0.48 & -0.49 & -0.32 & $-1.69 *$ & -0.02 & $1.18 * * *$ & 0.61 & -0.53 & -3.96 \\
\hline $\mathrm{P}_{1} \times \mathrm{P}_{3}$ & 1.05 & $-0.37 * * *$ & -0.26 & 2.09 & $0.69 * * *$ & -3.32 & $1.02 * * *$ & 0.59 & -0.56 & -1.89 & 0.11 & $5.19 *$ \\
\hline $\mathrm{P}_{1} \times \mathrm{P}_{4}$ & $5.06 * * *$ & $-0.47 * * *$ & -0.05 & $-3.43 * * *$ & -0.81 & $-1.04 * * *$ & 0.28 & -0.64 & 0.43 & -1.25 & $-0.39 * * *$ & $-6.36^{*}$ \\
\hline $\mathrm{P}_{1} \times \mathrm{P}_{5}$ & -0.18 & 0.6 & 0.19 & $-3.40 * * *$ & 0.04 & $2.80 *$ & -0.76 & -0.19 & 0.23 & 3.6 & -0.35 & -2.29 \\
\hline $\mathrm{P}_{1} \times \mathrm{P}_{6}$ & $2.37 * *$ & $0.96 * * *$ & 0.01 & 2.4 & $0.82 * * *$ & 3.4 & -0.18 & 1.28 & -1.78 & 3.5 & 0.24 & -7.58 \\
\hline $\mathrm{P}_{2} \times \mathrm{P}_{3}$ & $4.40 * * *$ & -0.23 & 0.05 & 0.73 & 1.15 & 1.26 & 0.56 & -0.68 & $0.58 * * *$ & 0.83 & 0.16 & 3.82 \\
\hline $\mathrm{P}_{2} \times \mathrm{P}_{4}$ & $5.37 * * *$ & $0.67 *$ & $-0.01 * * *$ & $1.07 * * *$ & $2.71 * * *$ & $-1.29 * * *$ & 0.56 & -0.61 & 0.15 & -0.28 & -0.3 & $-6.09 *$ \\
\hline $\mathrm{P}_{2} \times \mathrm{P}_{5}$ & -4.67 & -0.51 & $-0.26^{*}$ & 2.23 & 0.43 & $-2.75 * * *$ & -0.64 & 0.77 & $-1.97 *$ & -3.19 & 0.03 & 1.87 \\
\hline $\mathrm{P}_{2} \times \mathrm{P}_{6}$ & -10.47 & -0.35 & 0.2 & -1.62 & $1.27 * * *$ & -1.51 & $1.56^{* * *}$ & -2.34 & $1.08 * * *$ & -5.89 & $1.56 * * *$ & 20.86 \\
\hline $\mathrm{P}_{3} \times \mathrm{P}_{4}$ & 0.76 & $-1.00 *$ & -0.11 & $3.75 * * *$ & $1.19 * * *$ & -2.21 & 0.69 & -0.41 & $4.07 * * *$ & -0.69 & $0.65 * * *$ & $4.80 * * *$ \\
\hline $\mathrm{P}_{3} \times \mathrm{P}_{5}$ & 0.51 & $1.07 * * *$ & -0.04 & $-4.38 * *$ & $-0.95^{*}$ & $8.1 * * *$ & $-1.97 *$ & 0.09 & 2.2 & $5.14 * * *$ & $-1.14 * *$ & $-5.44 *$ \\
\hline $\mathrm{P}_{3} \times \mathrm{P}_{6}$ & 0.43 & 0.81 & -0.01 & 1.44 & -1.53 & 6.99 & 0.49 & -0.64 & $4.07 * * *$ & 1.94 & $-0.83 * * *$ & 9.2 \\
\hline $\mathrm{P}_{4} \times \mathrm{P}_{5}$ & -0.47 & -0.27 & 0.09 & 4.11 & $0.43 * *$ & $-2.64 * * *$ & $1.63 * * *$ & 0.61 & $1.39 * * *$ & $-0.77 * *$ & $1.03 * * *$ & 11.3 \\
\hline $\mathrm{P}_{5} \times \mathrm{P}_{6}$ & $-4.8 * * *$ & $2.2 * * *$ & -0.45 & $12.02 * * *$ & $-1.96^{* * *}$ & 10.91 & $5.54 * * *$ & 3.29 & -3.62 & $-3.19 * *$ & $1.69 * * *$ & 24.44 \\
\hline $\mathrm{P}_{4} \times \mathrm{P}_{6}$ & -7.91 & $2.42 * * *$ & 0.19 & $-8.7 * * *$ & 0.06 & $-2.63 * * *$ & -4.69 & -2.29 & $2.83 * * *$ & $11.97 * * *$ & $-1.6^{* * *}$ & -41.41 \\
\hline
\end{tabular}

Note. $*$ Significant at $\mathrm{P} \leq 0.05$, ** Significant at $\mathrm{P} \leq 0.01$ and $* * *$ Significant at $\mathrm{P} \leq 0.001, \mathrm{P}_{1}-$ NERICA $1, \mathrm{P}_{2}$ NERICA 2, $\mathrm{P}_{3}$ - NERICA 11, $\mathrm{P}_{4}$ - NERICA 15, $\mathrm{P}_{5}$ - SARO5, $\mathrm{P}_{6}$ - Komboka, SF - Spikelet fertility, LR - Leaf rolling, LD - Leaf drying, TGW - Thousand grain weight, GY - Grain yield; DTF - 50\% Days to flowering, Cc Chlorophyll content, PL - Panicle Length, NTL/H - Number of tillers per hill, DTM - Day to maturity, PW Panicle weight, PH - Plant Height.

\section{Discussion}

The analysis for variance of various genotypes in different environments (drought and non drought) in both sites showed significant differences for all traits evaluated implying appreciable amount of genetic variability of the germplasm used in the study. Thus, the genotypes evaluated can be selected for genetic improvement for grain yield and other agronomic traits under drought conditions. Previous researchers have emphasized the importance of genetic variation in the breeding of new improved varieties (Ismaila et al., 2013; Falconer, 1981). Parents NERICA 2 yielded high in both conditions while NERICA 15 had high yield potential and drought tolerance traits only under drought conditions. This was further confirmed by the GCA estimates in which for both parents they were positive for spikelet fertility and grain yields and negative for leaf rolling and drying. Previous studies reported breeding potential for parents based on mean performance and GCA effects (Muthuram et al., 2012; Rad et al., 2012). In contrast, SARO 5 and Komboka had low mean performance for physiological mechanism of drought tolerance such as spikelet fertility percentages, leaf rolling and leaf drying as well as yield contributing components such as a thousand grain weight and panicle weight. This was an indication that, the two parents were drought susceptible and they were affected by low soil water moisture hence, grain yields were affected drastically (Lafitte et al., 2003).

The concept of combining ability as introduced by Sprague and Tatum (1942) is the capacity of a parent to transmit superior performance to its crosses. In this study, parents with high mean performance and positive GCA are preferred for positive traits of grain yields under drought condition. On the other hand, parents with low estimates and negative GCA are suitable for negative traits of grain yield such as plant height, leaf rolling, leaf drying and days to $50 \%$ flowering.

The combining ability analysis revealed significant GCA and SCA variance for most of the traits under drought and non drought conditions suggesting the importance of both additive and non additive gene actions in expression of these traits. Further analysis of GCA/SCA predictability ratio (Baker, 1978) revealed that drought tolerance traits such as spikelets fertility, leaf rolling, leaf drying and chlorophyll content in this study were governed by non additive genes in addition to other yield contributing traits such as grain yield, thousand grain weight and number of tillers per panicle. Similar results were reported in previous studies (Priya, 2003; Yogameenaki et al., 2015; Subramanian et al., 1998). Hybridization followed by selection in later generations 
may be recommended for improvement of traits controlled by non additive gene actions. The relatively higher magnitude of GCA variance (fixable genetic components) indicated the predominant role of additive gene action for traits like $50 \%$ days to flowering (0.6 and 0.7), panicle length (0.6 and 0.8) in drought and non drought conditions respectively. Previous research reported similar results (Kumar et al., 2007; Yogameenakshi et al., 2015). Simple selection procedure and pedigree breeding are sufficient to improve traits controlled by additive gene actions (Lavanya, 2000; Muthuram et al., 2012). In this study, NERICA 2 and NERICA 15 showed good general combiners ability for drought trait parameters, panicle length and grain yield. Similar findings were previous reported by Kumar et al. (2008) and Yogameenakshi et al. (2015).

Specific combining ability effects give the usefulness of a particular cross combination. To select high yielding variety under drought environment, crosses with few days to flower, high percentages of spikelet fertility, more tillers number per hill and low score for leaf rolling/drying can be selected. Crosses NERICA $15 \times$ NERICA 2 , NERICA $1 \times$ NERICA 15 , NERICA $11 \times$ NERICA 15 and NERICA $2 \times$ NERICA 15 were top ranked for one or more drought tolerant trait(s) and yield components. All these crosses had either or shared one of the good parent combiner with drought tolerance traits signifying that these crosses will eventually yield desirable transgressive segregants (Zhang et al., 1994; Li et al., 2002; Alam et al., 2004).

The present study showed that, none of the parents or the specific crosses were the best general combiners for all drought traits was good combiner for all the traits evaluated. This implies that the parents used in this study were genetically diverse and can be selected for different traits for further improvement. Previous research has reported similar findings (Singh et al., 2007; Panwar et al., 2005; Sharma \& Mani, 2005).

Yield improvement in rice for drought prone environment is possible by selecting appropriate parents based on the mean performance and combining ability followed by suitable breeding programmes based on the nature of gene action involved (Muthuram et al., 2012; Yogameenakshi et al., 2015).

\section{Conclusions and Recommendations}

Drought tolerance traits namely the spikelets fertility, leaf rolling, leaf drying and chlorophyll content and the grain yield components like the grain yield, a thousand grain weight and number of tillers per panicle in this study were governed by non additive genes suggesting that hybridization followed by selection in later generations may be recommended for improvement of these traits. On the other hand, there was predominance of additive gene action effects among the traits such as plant height, panicle length and days to $50 \%$ flowering. In this study, two parents namely NERICA 2 and NERICA 15 were good combiners for drought traits, and grain yield components hence they could be utilized in hybridization program to introgress drought tolerance into elite lines. The crosses NERICA $15 \times$ NERICA 2, NERICA $1 \times$ NERICA 15, NERICA $11 \times$ NERICA 15 and NERICA $2 \times$ NERICA 15 also showed good SCA effects for one or more drought tolerant trait and yield components. Based on combining ability, none of the parents or specific crosses showed combination of all drought traits hence, to develop a drought tolerant genotype, a combination of desirable traits may be introgressed into adopted rice genotypes.

\section{Acknowledgements}

This publication was made possible through support provided by Alliance for a Green Revolution in Africa (AGRA). The authors also express sincere appreciation to Kenya Agricultural Livestock and Research Organization - Industrial Crop Research Center (ICRC) Mwea and JICA - Science and Technology Research Partnership for Sustainable Development (SATREPS) Project for provision of infrastructure for this study.

\section{References}

Alam, M. F., Khan, M. R., Nuruzzaman, M., Parvez, S., Swaraz, A. M., Alam, I., \& Ahsan, N. (2004). Genetic basis of heterosis and inbreeding depression in rice (Oryza sativa L.). Journal of Zhejiang University Science, 5(4), 406-411. https://doi.org/10.1631/jzus.2004.0406

Baker, R. J. (1978). Issues in diallel analysis. Crop Science, 18(4), 533-536. https://doi.org/10.2135/cropsci 1978.0011183X001800040001x

Boonjung, H., \& Fukai, S. (1996). Effect of soil water deficit at different growth stage on rice growth and yield under upland conditions, phenology, biomass production and yield. Field Crops Revision, 47-55. https://doi.org/10.1016/0378-4290(96)00039-1

Can, N. D., Nakamura, S., \& Yoshida, T. (1997). Combining ability and genotype $\times$ environment interaction in early maturing grain sorghum for summer seeding. Japan Journal of Crop Science, 66, 698-705. https://doi.org/10.1626/jcs.66.698 
Dar, S. H., Rather, A. G., Ahanger, M. A., \& Talib, S. (2014). Gene Action and Combining Ability Studies for yield and component traits in Rice (Oryza sativa L.): A Review. Journal of Plant and Pest Science, 1(3), 110-127.

Falconer, D. S. (1981). Introduction to quantitative genetics (2nd ed., Vol. 99, No. 3-4, pp. 541-553). New York: The Ronald Press Company. Retrieved from http://www.genetics.org/content/genetics/99/3-4/541

Gomez, K. A. (1972). Techniques for field experiments with rice. International Rice Research Institute (IRRI), Philippines. Retrieved from http://www.nal.usda.gov

Griffing, B. (1956a). Concept of general and specific combining ability in relation to diallel crossing systems. Australian Journal of Biological Sciences, 9(4), 463-493. https://doi.org/10.1071/BI9560463

Griffing, B. (1956b). A generalized treatment of the use of diallel crosses in quantitative inheritance. Heredity, 10, 31-50. https://doi.org/10.1038/hdy.1956.2

IRRI (International Rice Research Institute). (2014). Standard Evaluation System for Rice (SES) (5th ed.). Los Banos, Philippines.

Ismaila, A., \& Echekwe, C. A. (2012). Genetics of some Agronomic and Yield Traits in rice (Oryza sativa L.). International Journal of Science and Research, 1, 1311-1322.

Kempthorne, O. (1957). An Introduction to Genetic Statistics (p. 545). John Wiley and Sons, Inc: New York. Retrieved from http://psycnet.apa.org/psycinfo/1958-01083-000

KEPHIS. (2015). Kenya National Crop Variety List. Retrieved from http://www.kephis.org

Kimani, J. M. (2010). Genetic studies of quantitative and quality traits in rice under low and high soil nitrogen and phosphorous conditions, and a survey of farmer preferences for varieties $(\mathrm{PhD}$ thesis, University of KwaZulu-Natal, Republic of South Africa).

Kobayashi, S., Fukuta, Y., Sato, T., Osaki, M., \& Khush, G. S. (2003). Molecular marker dissection of rice (Oryza sativa L.) plant architecture under temperate and tropical climates. Theoretical and Applied Genetics, 107(8), 1350-1356. https://doi.org/10.1007/s00122-003-1388-8

Kumar, A., Bernier, J., Verulkar, S., Lafitte, H. R., \& Atlin, G. N. (2008). Breeding for drought tolerance: Direct selection for yield, response to selection and use of drought-tolerant donors in upland and lowland-adapted populations. Field Crops Research, 107(3), 221-231. https://doi.org/10.1016/j.fcr.2008. 02.007

Kumar, A., Dixit, S., Ram, T., Yadaw, R. B., Mishra, K. K., \& Mandal, N. P. (2014). Breeding high-yielding drought-tolerant rice: Genetic variations and conventional and molecular approaches. Journal of Experimental Botany, 107(3), 221-231. https://doi.org/10.1016/j.fcr.2008.02.007

Kumar, K., \& Pandey, M. P. (2008). Combining ability and heterosis over environments for yield and yield components in two-line hybrids involving thermo sensitive genic male sterile lines in rice (Oryza sativa L.). Plant Breeding, 127(1), 28-32.

Kumar, S., Singh, H. B., \& Sharma, J. K. (2007). Combining ability analysis for grain yield and other associated traits in rice (Oryza sativa). International Journal on Rice, 44(2), 108-114. Retrieved from http://www.indianjournals.com

Lafitte, R., Blum, A., \& Atlin, G. (2003). Using secondary traits to help identify drought-tolerant genotypes. Breeding rice for drought-prone environments (pp. 37-48). International Rice Research Centre (IRRI), Los Bańos, Philippines. Retrieved from http://books.irri.org/9712201899

Li, W., Zhang, J. Z., Zhang, G. Q., \& Zuo, Q. F. (2002). Analysis of heterosis of main agronomic traits in indica-japonica lines of rice. Journal of Southwest Agriculture University, 24(4), 317-320.

Menge, D. M., \& Makihara, D. (2013). Output on rice research in Kenya (pp. 1-2).

Muthuram, S., Jebaraj, S., \& Gnanasekaran, M. (2010). Combining ability and heterosis for drought tolerance in different locations in rice (Oryza sativa L.). Journal of Agricultural Science, 1, 266-270.

Panwar, L. L. (2005). Line $\times$ tester analysis of combining ability in rice (Oryza sativa L.). The Indian Journal of Genetics and Plant Breeding, 65(1), 51-52.

Peyman, S., Motlagh, M. R. S., \& Aminpanah, H. (2012). Diallel analysis for salinity tolerance in rice traits at germination stage. African Journal of Biotechnology, 11(14), 3276. 
Priya, P. A. (2003). Genetic analysis of drought tolerance and yield components in rice (Oryza sativa L.). Electronic Journal of Plant Breeding, 2(1), 24-30.

Rad, M. R. N., Kadir, M. A., Yusop, M. R., Jaafar, H. Z., \& Danaee, M. (2012). Combining ability and inbreeding depression of physiological parameters in F. Journal of Food, Agriculture \& Environment, 10(3\&4), 875-878.

Samonte, S. O., Wilson, L. T., McClung, A. M., \& Tarpley, L. (2001). Seasonal dynamics of non-structural carbohydrate partitioning in 15 diverse rice genotypes. Crop Science, 41(3), 902-909. https://doi.org/ 10.2135/cropsci2001.413902x

Sharma, R. K., \& Mani, S. C. (2005). Combining ability and genes action for quality character in Basmati Rice. Indian Journal of Genetics, 65(2), 123-124

Sprague, G. F., \& Tatum, L. A. (1942). General vs. specific combining ability in single crosses of corn. Agronomy Journal, 34(10), 923-932. https://doi.org/10.2134/agronj1942.00021962003400100008x

Subramanian, J., Rangasamy, P., Vaidyanathan, P., \& Thangaraj, M. (1998). Combining ability for drought resistant characters in hybrid rice (Oryza sativa). Indian Journal of Agricultural Science, 68(10), 687-689. Retrieved from http://citeseerx.ist.psu.edu/viewdoc/download?doi=10.1.1.177.6908

Suresh, R., Chandra, R. B., Gomez, M., \& Shanmugasundaram, P. (2013). Genetic analysis of yield traits in rice under irrigated and water stress environment. Indian Journal of Genetics, 73(2), 162-168. https://doi.org/10.5958/j.0975-6906.73.2.023

Torres, E. A., \& Geraldi, I. O. (2007). Partial diallel analysis of agronomic characters in rice (Oryza sativa L.). Genetics and Molecular Biology, 30(3), 605-613. https://doi.org/10.1590/S1415-47572007000400018

Verma, O. P. (2003). Diallel analysis in rice (Oryza sativa L.) for physiological traits. Madras Agricultural Journal, 90, 637-642.

Verulkar, S., Mandal, N., Dwivedi, J., Singh, B., Sinha, P., Mahato, R., ...Swain, P. (2010). Breeding resilient and productive genotypes adapted to drought-prone rainfed ecosystem of India. Field Crops Research, 117, 197-208. https://doi.org/10.1016/j.fcr.2010.03.005

Yogameenakshi, P., \& Vivekanandan, P. (2015). Combining ability analysis for drought tolerance in rice (Oryza sativa L.) under reproductive stage. Electronic Journal of Plant Breeding, 6(2), 373-381. Retrieved from http://www.indianjournals.com/ijor.aspx

Zhang, Q., Gao, Y. J., Yang, S. H., Ragab, R. A., Maroof, M. S., \& Li, Z. B. (1994). A diallel analysis of heterosis in elite hybrid rice based on RFLPs and microsatellites. Theoretical and Applied Genetics, 89(2-3), 185-192.

Zou, G. H., Mei, H. W., Liu, H. Y., Liu, G. L., Hu, S. P., Yu, X. Q., \& Luo, L. J. (2005). Grain yield responses to moisture regimes in a rice population: Association among traits and genetic markers. Theoretical and Applied Genetics, 112(1), 106-113.

\section{Copyrights}

Copyright for this article is retained by the author(s), with first publication rights granted to the journal.

This is an open-access article distributed under the terms and conditions of the Creative Commons Attribution license (http://creativecommons.org/licenses/by/4.0/). 